Letter to the Editor

\title{
Alcohol mixed with energy drinks and arrhythmias
}

\section{Dear Editor,}

We have read with great interest the paper "Nature versus intensity of intoxication: Co-ingestion of alcohol and energy drinks and the effect on objective and subjective intoxication" by Forward and coworkers (Forward et al., 2017) and we found their conclusion of importance with a view to clinical prevention for young people. Authors reported studies on the effect of alcohol mixed with energy drinks (AmEDs), versus alcohol alone, examining objective intoxication, intensity, and nature of intoxication.

They concluded that reductions in objective intoxication and perceived intensity of intoxication, but not nature of intoxication, were observed after AmED consumption.

With reference to this paper, we would like to make the following contribution to the discussion. Episodes of atrial fibrillation (AF) in young people after ingestion of commercial energy drinks have been reported. (Mattioli et al., 2017). We hypothesized that the combined effect of caffeine and other substances included in energy drinks (i.e. taurine) can trigger the onset of arrhythmia. The relationship between caffeine and arrhythmias is controversial. Because of its stimulant effect, there is concern that caffeine might increase arrhythmic risk. However, it is known that moderate doses of caffeine are well tolerated in patients with arrhythmia, and that the exposure to low doses of caffeine might offer protective effect against AF. (Caldeira et al., 2013; Mattioli 2007; Mattioli et al., 2011). Little information is available on the link between EDs and arrhythmias (De Sanctis et al., 2017; Mattioli et al., 2017). Recently we observed 2 cases of atrial fibrillation in young subjects after acute ingestion of EDs mixed with alcohol. Patients presented with palpitations. In both cases arrhythmia recovered after pharmacological cardioversion. ECG before cardioversion showed arrhythmia with high ventricular rate, while ECG after cardioversion showed a sinus tachycardia and the heart rate spontaneously reduced after 3-5h. No other abnormalities were reported in the ECG. It is possible that caffeine acts as a trigger for the development of arrhythmias by increasing sympathetic activity. (Mattioli, 2007). Energy drinks included high dosage of caffeine and many other substances known to have caffeinelike effects (ie. Guaranà) and other cardiac effects (De Sanctis et al., 2017; Mattioli et al., 2005). However, despite the widespread tendency of drinking AmED among young people, episodes of arrhythmias have been rarely reported This might be attributable to the fact that some episodes of atrial fibrillation are asymptomatic, and that alcohol can reduce the perception of arrhythmia as suggested by Forward and coworkers. They confirm the level of intoxication and impairment was rated lower after consuming AmED as compared to alcohol alone. (Forward et al., 2017)

Furthermore, it is plausible that only a sub-group of subjects is susceptible to developed arrhythmias during binge ingestion of EDs and alcohol. Alcohol can increase side effects of EDs through unknown mechanisms.

We support the hypothesis from Forward that psychological (e.g., beverage expectancy effects), biological (e.g., other carbohydrate consumption, sex), and environmental factors (e.g., drinking peers) influence objective and subjective intoxication in the real world. (Forward et al., 2017). This needs to be investigated to identify high-risk subjects.

In conclusion, we agree with Forward and coworkers that larger clinical trials, evaluating the effects of alcohol mixed with energy drinks on cerebral and cardiovascular systems, are warranted.

\section{Authors disclosure}

No conflict of interest.

\section{List of contributors}

Anna Vittoria Mattioli, Antonio Manenti, Alberto Farinetti.

\section{References}


De Sanctis, V., Soliman, N., Soliman, A.T., Elsedfy, H., Di Maio, S., El Kholy, M., Fiscina, B., 2017. Caffeinated energy drink consumption among adolescents and potential health consequences associated with their use: a significant public health hazard. Acta Biomed. 88, 222-231.

\footnotetext{
DOI of original article: http://dx.doi.org/10.1016/j.drugalcdep.2018.02.006

Letter related to "Forward J., Akhurst J., Bruno R., Leong X., VanderNiet A., Bromfield H., Erny J., Bellamy T., Peacock A., Nature versus intensity of intoxication: Co-ingestion of

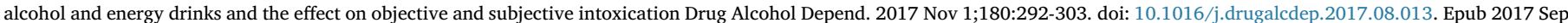
7."
} 


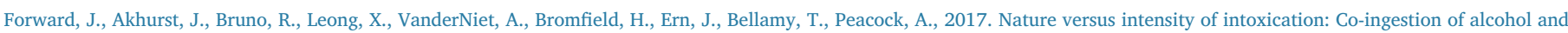
energy drinks and the effect on objective and subjective intoxication. Drug Alcohol Depend, 180, 292-303.

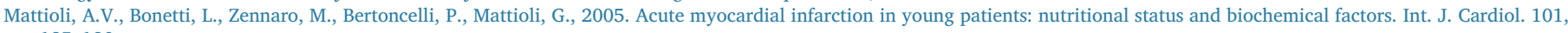
$185-190$.

Mattioli, A.V., Farinetti, A., Miloro, C., Pedrazzi, P., Mattioli, G., 2011. Influence of coffee and caffeine consumption on atrial fibrillation in hypertensive patients. Nutr. Metab. Cardiovasc. Dis. 21, 412-417.

Mattioli, A.V., Pennella, S., Farinetti, A., Manenti, A., 2017. Energy drinks and atrial fibrillation in young adults. Clin. Nutr. http://dx.doi.org/10.1016/j.clnu.2017.05.002.

Mattioli, A.V., 2007. Effects of caffeine and coffee consumption on cardiovascular disease and risk factors. Future Cardiol. 3, $203-212$.

Anna Vittoria Mattioli*, Antonio Manenti, Alberto Farinetti Surgical, Medical and Dental Department of Morphological Sciences Related to Transplant, Oncology and Regenerative Medicine, University of Modena and Reggio Emilia, Italy E-mail address: annavittoria.mattioli@unimore.it 